\title{
A shooting algorithm based on Angular Bisector Path Planning
}

\author{
Yang Jiangping \\ North China Electric Power University (Baoding) \\ Baoding, Hebei, China \\ yangjiangping321@126.com
}

Liu Li

North China Electric Power University (Baoding) Baoding, Hebei, China

\begin{abstract}
In order to improve the Underwater Robot's probability of success of the shot and the speed of the shot in water polo competitions, a shooting algorithm based on Angular Bisector Path Planning using geometric method was proposed. The algorithm computes rapidly and shoots in high efficiency. The algorithm implementation was given and tested on the simulation platform. The effects were also evaluated. The algorithm has been successfully applied in actual competitions. The results proved that has a rapid offensive and strong interference suppression. The feasibility and effectiveness of the algorithm was verified by the experiment.
\end{abstract}

Keywords—robot fish;Angular Bisector;path planning; shot

\section{INTRODUCTION}

For the past few years, the Robot Technology is developing rapidly. It takes the Biomimetic Robot Fish as the subject in the robot polo competitions, which is similar with the match play about the robot soccer match on land ${ }^{[1]}$. The match not only involved all kinds of technologies on Robot Soccer Applications, but also referred to many fields such as Hydrodynamic analysis and anti-jamming technology and so on ${ }^{[2]}$. There are some technical challenges, as well as enjoyment. Thus, it's a perfect combination of scientific research and science ${ }^{[3]}$.

When the two teams confront with each other in robot fish match, one team wins if it shoots water polo into the opponent's goal more times than the other team during a limited time. And the key to triumph is shooting ${ }^{[4]}$. Pros and cons of shooting algorithm and strategies to deal with special circumstances will influence the probability of success directly. For robot fish in water polo competitions, a new shot algorithm based on bisector path planning was proposed. The simu lation platform's name is URWPGSim2D, which is developed by Peking University. The algorithm obtains a set of robot fish's temporary target points. It is just making robot fish, water polo and target point in a straight line. And then the robot fish gets to the point of head polo. The fish can easily take the water polo into the opponent's goal. The algorithm improves the efficiency of shooting greatly.

This research was financially sponsored by "the Fundamental Research Funds for the Central Universities" (No.2014MS1 28)", "North China Electric Power University (Baoding) Innovation Training Project (No.20140053)" and "North China Electric Power University 2014 Undergraduate Education Reform Project".

\author{
Song Xuhe \\ North China Electric Power University (Baoding) \\ Baoding, Hebei, China \\ 2591439071@qq.com
}

\section{INTRODUCTION OF ROBOT FISH}

Taking the simulate robot fish as the research object on the simulation platform, The fish's structure, size, color and motion parameters of four aspects are described as following, which is according to the definition of the robotic fish's $2 \mathrm{D}$ model.

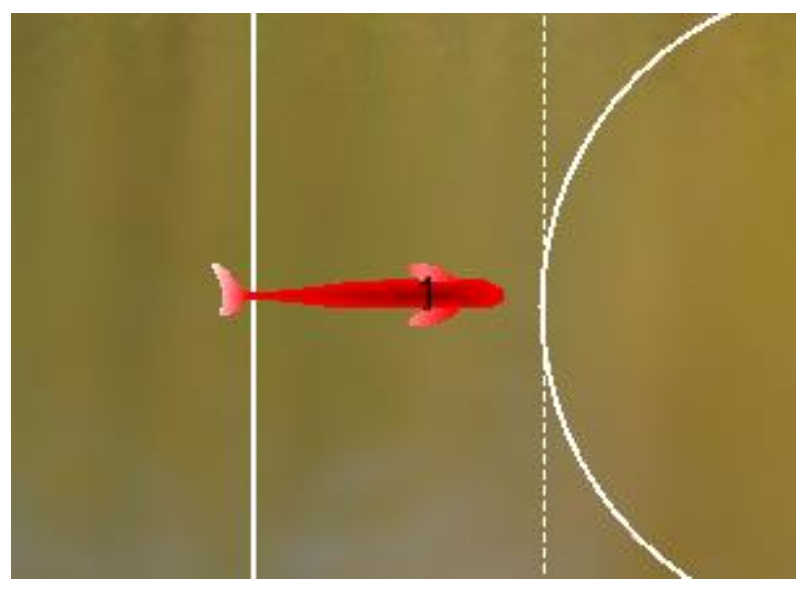

Figure 1. The Robot Fish

As shown in Figure 1, the black digital on robot fish body indicates its order in its own team. The robot fish parameters are designed as the entity fish.

\section{A. Structure}

The fish's shape consists of three parts. It includes a curved head, a rectangular body, an elongated rectangular tail fin and two pectoral fins in right triangle. And the body is divided into three small parts. Each small part is isosceles trapezoid. And the bottom edge of the length of isosceles trapezoid is diminishing.

\section{B. Size}

The radius of arc head is 22 millimeter, rectangle of fish about Length * Width is $160 \mathrm{~mm} * 45 \mathrm{~mm}$. Three bottom edge's length of is osceles trapezoid are $45 \mathrm{~mm}, 30 \mathrm{~mm}$ and 
$18 \mathrm{~mm}$. The height of the three trapezoid are $88 \mathrm{~mm}, 66 \mathrm{~mm}$ and $55 \mathrm{~mm}$.

\section{Color}

Robot fish color of the rectangle can be configured by the platform module. Different teams have their own color.

\section{Motion Parameters}

The robot fish have 15 kinds of speed gears and direction gears. Speed setting higher the value, the faster of the robot fish's movement speed it is. The direction gears are divided into 3 parts. 0 6 represent robot fish turn left, 7-speed represent robot fish swim straightly and 8 to 14 gears represent robot fish turn right.

\section{A SHOOTINGALGORITHM BASED ON ANGULAR BISECTOR PATH PLANNING}

Angular Bisector Path Planning conforms the robot fish's trajectory according to the points of fish, water polo and the goal. When the connection line of fish and water polo is not horizontal, robot fish use the algorithm to adjust its direction continuously in the process of arrival at water polo. The fish's orientation is just the same as the direction of connection line of the ball and the goal when fish just arrives at the head point of water polo. This would ensure that the fish can reach the head point within a short time to head the water polo, thereby increasing the efficiency of shot.

\section{A. Conditions for the Algorithm}

(1) Requirements for selecting target

The simulation platform's default target is the center of the goal. As shown in Figure 2, the target is the point of $G_{0}$, and in order to augment shooting angle, the position of the target point $G_{0}$ could be adjusted dynamically. The point $G_{0}$ can be changed with the changing of water polo's position. Target point could be located in a position that above the central point, namely point $G_{1}$ and the Z-axis coordinate value is $Z g=-\sigma$ when water polo's $Z$-axis coordinate (just below the platform is the Z-axis) is greater than zero. Similarly, Target point also could be located in a position that below the central point, namely point $G_{2}$ and the Z-axis coordinate value is $Z g=+\sigma$ when water polo's $Z$-axis coordinate is less than zero. Setting $\sigma=100 \mathrm{~mm}$ based on experience.

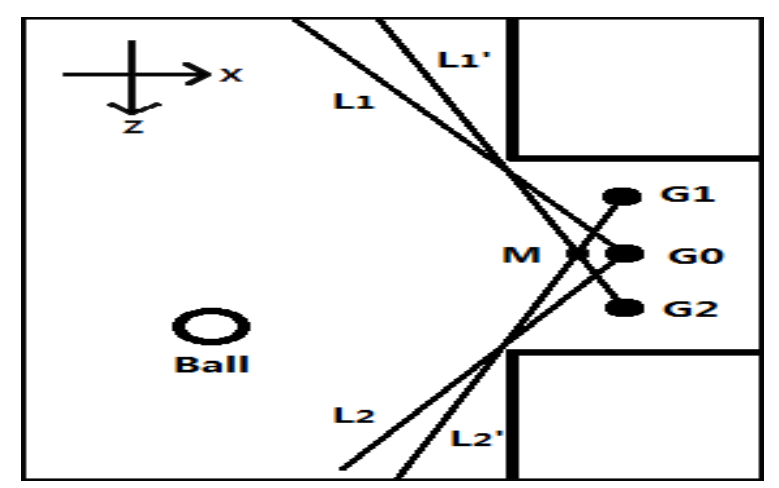

Figure 2. Selecting the target
Target set in the region of goal are between point $G_{1}$ and point $G_{2}$. It has a bigger angular of shooting than that selecting of the target position for the center of the goal simply. Shooting angle is the angle formed by line $L_{1} G_{0}$ and line $L_{2} G_{0}$ when target at the center of goal. However, the shooting angle would increase to that formed by line $L_{1}^{\prime} M$ and line $L_{2}^{\prime} M$. Thus, the probability of success of shooting would increase obviously.

(2) Requirements for shooting distance

Own goals often happens in the actual match. There are two main reasons. One happens when two teams scramble for the water polo. The other happens when fish shoot in an improper playing area. Although the target point is not that is its own goal, the robot fish does curve movement might take water polo into one's own goal. For the two reasons described above, two restrictions are set as following.

- Water polo should have a distance that in the range of $300 \mathrm{~mm} 500 \mathrm{~mm}$ from the target point.

- Robot fish should be within the scope of the circu mference to the water polo center, at a distance $d$ (typically take $700 \mathrm{~mm} 900 \mathrm{~mm}$ ) as the radius.

These two restrictions ensures that robot fish could be in the vicinity of the opponent's goal when shooting at goal.

\section{B. Description of the algorithm}

In the simulation platform of competition area, robot fish needs to take water polo into the target point Goal. As shown in Figure 3, the default coordinate system of the simulation platform is $X-Z$ coordinate system, which is shown on the upper right corner.

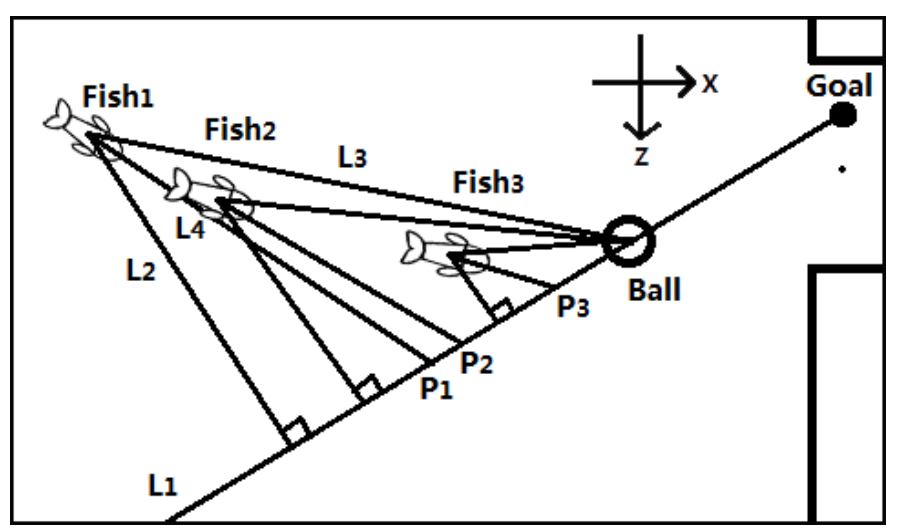

Figure 3 . The shooting algorithm based on angular bisector path planning

Specifics steps of the algorithm is as follows:

Step 1: Make a connection between point Goal and point Ball. And make its reversely extension cord, named $L_{1}$. 
Step 2: Cross the fish and make the vertical line $L_{2}$ of line $L_{1}$, then make a connection line $L_{3}$ between

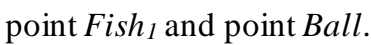

Step 3: Make the angular bisector line $L_{4}$ which is between line $L_{2}$ and line $L_{3}$. And the intersection of line $L_{1}$ and line $L_{4}$ is referred to as point $P_{1}$.

Step 4: Robot fish takes point $P_{1}$ as the interim target point to s wim.

Step 5: If the fish reaches the position of water polo, then it just head the ball forward, otherwise, go Step 1 mentioned above.

Robot fish calls the above algorithm constantly during the motion. The interim target point changes to be point $P_{2}$ when robot fish reaches at point $\mathrm{Fish}_{2}$. And the interim target point also changes to be point $P_{3}$ when robot fish reaches at point Fish 3 . The fish's orientation is just agree with the direction of connection line of the ball and the goal, namely line $L_{1}$, when fish just arrives at the head point of water polo. And the position is very favorable shot for the robot fish.

Note that robot fish's position changes constantly and the water polo's might also changes continuously during the process of motion. Therefore, robot fish should call the above algorithm to adjust its path continually during every refresh cycle.

\section{Design Parameters}

- Mathematical Symbols

The meaning of model mathe matical symbols is shown in Table 1.

TABLE I.

SYMBOLS AND DEFINITIONS

\begin{tabular}{c|c}
\hline Symbols & Notations \\
\hline$k_{i}$ & The slope of straight line $i$ \\
$\theta_{i}$ & $\begin{array}{c}\text { The angle between the straight line } i \text { and } X- \\
\text { axis positive direction }\end{array}$ \\
$X_{j}$ & The value of $X$ coordinate of point $j$ \\
$Z_{j}$ & The value of $Z$ coordinate of point $j$ \\
\hline
\end{tabular}

\section{- Equations}

The key to algorithm solution is to find the angle between line $L_{4}$ and $X$-axis. Although the point Goal's coordinate depends on water polo's value of $Z$ coordinate. However, the ball's position Ball $\left(x_{b}, z_{b}\right)$ could be obtained real-time in the global visual simulation environment.
Thereby, the point Goal $\left(x_{g}, z_{g}\right)$ can be confirmed. Assuming the slope of line $L_{1}$ is $k_{1}$, as in

$$
k_{1}=\frac{z_{g}-z_{b}}{x_{g}-x_{b}}
$$

Line $L_{1}$ is vertical with line $L_{2}$, so the slope of line $L_{2}$ is $k_{2}$, as in

$$
k_{2}=-\frac{1}{k_{1}}
$$

The fish's position Fish $\left(x_{f}, z_{f}\right)$ also can obtain, so the slope of line $L_{3}$ is $k_{3}$, as in

$$
k_{3}=\frac{z_{f}-z_{b}}{x_{f}-x_{b}}
$$

$\theta_{2}$ And $\theta_{3}$ could calculate by equation (2) and (3).

The angle between the line $\mathrm{L} 2$ and $\mathrm{X}$-axis is $\theta_{2}$, as in

$$
\theta_{2}=\arctan k_{2}
$$

The angle between the line $\mathrm{L} 3$ and $\mathrm{X}$-axis is $\theta_{3}$, as in

$$
\theta_{3}=\arctan k_{3}
$$

The angle between the line L4 and $\mathrm{X}$-axis is $\theta_{4}$, as in

$$
\theta_{4}=\min \left\{\theta_{2}, \theta_{3}\right\}+\frac{\left|\theta_{2}-\theta_{3}\right|}{2}
$$

According to these six mathematical formulas, the robot fish's shooting algorithm can be implemented using C\# language in .Net Programming environment.

\section{SimUlATION AND ANALYSIS}

Based on URWPGSim2D simulation platform, the motion track of robot fish is shown in Figure 4. Firstly, the robot fish use the shooting algorithm based on angular bisector path planning move along the curve line $L_{1}$. Then it head the water ball directly into the goal along with line $L_{2}$. 


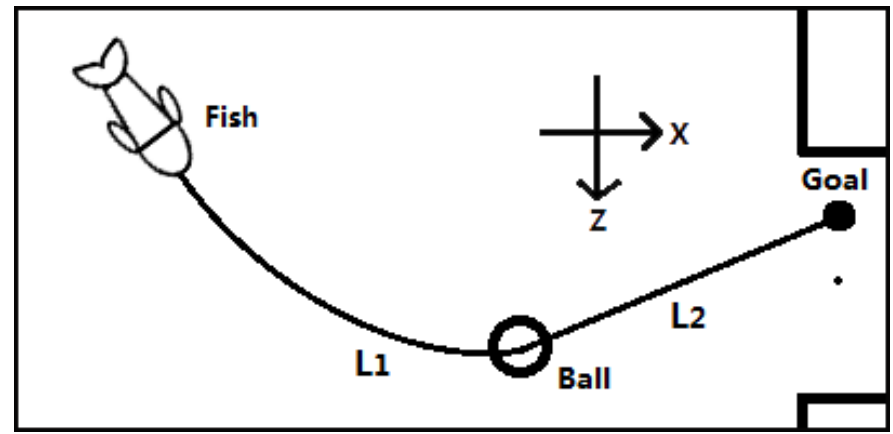

Figure 4. Motion path

After many simulation tests, it is found that there is a high probability of scoring using the shooting algorithm based on angular bisector path planning. And the algorithm is simple and efficient.

Its advantages were summarized as the following:

- In the process of reach the point of ball movement, the robot fish could adjust their own direction constantly. There is a high efficiency to shoot the goal along a straight line directly after it reached the ball point.

- The algorithm could be used in the situation that the distance is great.

- By adding the constraints of shooting, the own goal is prevented effectively.

- The angle of shot options are expanded, and the chance of shooting is increased indirectly.

- By solving several simple mathematical formula, the runtime of algorith $\mathrm{m}$ is constant magnitude. And it has a high running speed.

The algorithm also has shortcomings. When the fish is located in the middle of ball and the target point, it will waste a lot of time to look for angle bisector and the target point and the ball attachment reverse extension point. The shooting efficiency will be reduced.

\section{CONCLUSIONS}

A new shooting algorithm based on angular bisector path planning of shot is proposed. The main method is to adjust its direction constantly in the process of moving. Meanwhile, fish body's direction to keep the ball and the direction of approaching the target point connections, making it possible to begin to head the ball to travel along a straight line to head the ball points. The algorithm has been applied to the simulation platform match scene 3 VS 3, and the good results are obtained. It could be applied in ball big battle and water polo events in the snooker. This algorithm is used in addition to the main underwater robot fish, it could also be used for 3D robot soccergame shooting.

\section{ACKNOWLEDGMENT}

This research was financially supported by "the Fundamental Research Funds for the Central Universities" (No.2014MS 128)", "North China Electric Power University (Baoding) Innovation Training Project (No.20140053)" and
"North China Electric Power University 2014 Undergraduate Education Reform Project".

\section{REFERENCES}

[1] Liu Hongzhi, “An improved shooting algorithm". Harbin Institute of Technology Learned Journal, 2004,36 (7) : 975-977

[2] Tao Jin, Kong Feng and Xie Guangming. "Behavior-Based Motion Planning of Bioimet ic Robot-Fish'[J]. Ordnance Industry Aut omation, 2010,11:70-73.

[3] Han Xuedong, Hong Bingrong, and Meng Wei. "Shooting algorithm in robot soccer" [J]. Harbin Institute of Technology Leamed Journal, 2003, 09: 1064-1066.

[4] Tan Min, Zhang Xin, T ang Lin, and Deng Yansong. "Study on the 3 vs 3 Strategies of Emulation of Robotic Fish". College of Electrical and Information Engineering, Southwest University for Nationalities, 2011,37(5): 231-234.

[5] Xie Chaoping. "Path Planning of Biomimetic Robotic Fish". Guangxi Institue of Technology, 2010.

[6] Xiao Quan, Kong Feng, and Tao Jin. "Research on Path Optimization of Point Fuzzy Control on Biomimetic Robot fish". Department of Electronic Information and Control Engineering, 2011.

[7] Chen Wei. "The Research of Locomotion Modal and Game Strategy of Robot-Fish'[D]. Guangxi Institue of T echnology, 2012. 